\title{
Gluteus medius muscle function in people with and without low back pain: a systematic review
}

\author{
Sean Sadler ${ }^{1 *}$ D, Samuel Cassidy ${ }^{1}$, Benjamin Peterson ${ }^{1}$, Martin Spink ${ }^{1,2}$ and Vivienne Chuter ${ }^{1,2}$
}

\begin{abstract}
Introduction: Globally, low back pain (LBP) is one of the greatest causes of disability. In people with LBP, dysfunction of muscles such as the gluteus medius have been demonstrated to increase spinal loading and reduce spinal stability. Differences in gluteus medius function have been reported in those with LBP compared to those without, although this has only been reported in individual studies. The aim of this systematic review was to determine if adults with a history, or current LBP, demonstrate differences in measures of gluteus medius function when compared to adults without LBP.

Methods: MEDLINE, EMBASE, AMED, PsycINFO, PubMED, Pro Quest Database, CINAHL and SPORTDiscus were searched from inception until December 2018 for published journal articles and conference abstracts. No language restrictions were applied. Only case-control studies with participants 18 years and over were included. Participants could have had any type and duration of LBP. Studies could have assessed gluteus medius function with any quantifiable clinical assessment or measurement tool, with the participant non-weight bearing or weight bearing, and during static or dynamic activity. Quality appraisal and data extraction were independently performed by two authors.
\end{abstract}

Results: The 24 included articles involved 1088 participants with LBP and 998 without LBP. The gluteus medius muscle in participants with LBP tended to demonstrate reduced strength and more trigger points compared to the gluteus medius muscle of those without LBP. The level of activity, fatigability, time to activate, time to peak activation, cross sectional area, and muscle thickness showed unclear results. Meta-analysis was not performed due to the heterogeneity of included studies.

Conclusion: Clinically, the findings from this systematic review should be considered when assessing and managing patients with LBP. Future studies that clearly define the type and duration of LBP, and prospectively assess gluteus medius muscle function in those with and without LBP are needed.

Trial registration: PROSPERO (CRD42017076773).

Keywords: Low back pain, Systematic review, Gluteus medius, Electromyography

\footnotetext{
* Correspondence: sean.sadler@newcastle.edu.au

'Discipline of Podiatry, University of Newcastle, Ourimbah, NSW 2258,

Australia

Full list of author information is available at the end of the article
}

(c) The Author(s). 2019 Open Access This article is distributed under the terms of the Creative Commons Attribution 4.0 International License (http://creativecommons.org/licenses/by/4.0/), which permits unrestricted use, distribution, and reproduction in any medium, provided you give appropriate credit to the original author(s) and the source, provide a link to the Creative Commons license, and indicate if changes were made. The Creative Commons Public Domain Dedication waiver (http://creativecommons.org/publicdomain/zero/1.0/) applies to the data made available in this article, unless otherwise stated. 


\section{Introduction}

Low back pain (LBP) has been identified as the leading contributor of disability and was ranked sixth largest contributor to the burden of global disease, costing individuals and governments billions of dollars in both direct and indirect costs annually [1]. The prevalence of LBP increases linearly after the third decade of life [2], and, with an ageing population, the prevalence and impact of this condition are expected to increase [1].

Dysfunction of muscles of the lumbopelvic-hip complex is a hallmark of LBP [3]. At the hip, individuals with LBP are more likely to exhibit reduced gluteus medius muscle strength [4], reduced hip abduction force output [5], and altered hip muscle recruitment, demonstrating a distal-to-proximal muscle activation pattern in the lower limb compared to proximal-todistal in healthy controls [6]. These alterations to gluteus medius muscle function and strength have been suggested to lead to LBP [7], however, it is unknown whether such muscle deconditioning or atrophy is the cause or result of symptomatic LBP.

The gluteus medius is one of the main pelvic stabiliser muscles and plays a significant role in controlling transverse and frontal plane motion of the femur and hip [8], providing stability to the lumbopelvic-hip complex [9]. This stability may be important in controlling excessive movement and allowing adequate attenuation of forces throughout the lower back region. Gluteus medius weakness and consequential loss of dynamic lateral stability of the pelvis and lower back is suggested to lead to increased lateral trunk flexion and subsequent intervertebral disc compression [10], as well as altered movement patterns which may contribute to the development or exacerbation of LBP during standing [11-15].

Individual studies have found differences in the activation, strength, and number of trigger points in the gluteus medius muscle between those with and without LBP $[12,16-18]$. Due to these differences in gluteus medius muscle function, perhaps this muscle has a role in either the development or exacerbation of LBP. The mechanism by which this occurs is suggested to relate to the role in which the gluteus medius muscle plays in providing both frontal and transverse plane stability of the pelvis and lower back [13-15]. Determining the nature of gluteus medius function in those with LBP compared to those without is a key component to more effective assessment techniques and management of the condition. Therefore, a systematic review that collectively evaluates gluteus medius function in those with and without LBP is required.

This systematic review aims to determine, by review of case-control studies, if adults with a history of, or current LBP, demonstrate differences in measures of gluteus medius function when compared to adults without LBP. A secondary aim is to investigate if there is a difference in gluteus medius muscle function between types and durations of LBP.

\section{Methods \\ Search strategy}

This systematic review was registered with PROSPERO (CRD42017076773) and has been reported in accordance with the PRISMA statement [19]. MEDLINE, EMBASE, AMED, PsycINFO, PubMED, Pro Quest Database, CINAHL and SPORTDiscus were searched from inception until 14th December, 2018. No language restrictions were applied to published articles or conference abstracts. Keywords were truncated and combined using AND/OR, with search terms adapted for each of the databases (Additional file 1).

\section{Eligibility criteria}

Case-control studies including participants 18 years and older with LBP of any type (specific or non-specific) and of any duration (acute, subacute or chronic) were eligible for inclusion. Studies measuring gluteus medius function in any way, for example, strength, flexibility, fatigability, percentage of maximum voluntary contraction, crosssectional area, timing or extent of contraction, or other unidentified measurement were eligible for inclusion. Studies could assess gluteus medius with any quantifiable clinical assessment or measurement tool, with the participant non-weight bearing or weight bearing, and during static or dynamic activity.

Studies were excluded if they included participants that were pregnant, had a history of low back surgery, or were solely investigating the effect of an intervention on the gluteus medius muscle.

\section{Study selection}

One reviewer conducted the electronic searches (SS). Two reviewers (SC/SS) independently screened citations at title and abstract level. One reviewer (SS) retrieved potentially eligible full text articles and these were assessed independently by two reviewers (SC and SS). Authors were contacted where clarification was required for assessing eligibility for inclusion. There were no disagreements so there was no need to seek arbitration by a third reviewer (VC). Data were independently extracted by two reviewers (SC and $\mathrm{BP}$ ), using a standardised data extraction form, and cross checked by a third reviewer (SS). For the purposes of study classification we defined duration of back pain as: Acute ( $<6$ weeks), subacute (6- $<12$ weeks), and chronic ( $\geq 12$ weeks) [20]. Due to the heterogeneity between studies, a meta-analysis was not performed. 


\section{Quality assessment}

Two reviewers $(\mathrm{SC} / \mathrm{BP})$ independently appraised eligible full text articles using the CASP tool for casecontrol studies. The results of quality appraisal were checked by a third reviewer (SS) and no disagreements occurred.

\section{Results}

\section{Study identification}

Searches retrieved 1942 citations of which 94 were eligible for full text review. After review, 24 full text articles of mixed methodological quality (Additional file 2) were included, while 70 were excluded (Additional file 3) based on exclusion criteria (Fig. 1).

After contacting authors for eligibility confirmation, one conference abstract was excluded as it was confirmed it duplicated published journal article data [5]. Two full text articles could not be retrieved and were not provided by authors $[21,22]$ so were subsequently excluded.

\section{Characteristics of included studies}

The 24 articles [3-6, 16-18, 23-39] investigating gluteus medius function included 1088 participants with LBP and 998 healthy controls without LBP (Table 1). Studies included one or more of the following gluteus medius measurement outcomes: electromyographic (EMG) activity level [17, 18, 26, 28, 29, 31, 34, 38], EMG fatigability $[24,31,34]$, EMG time to onset [6, 26, 29, 30], EMG time of peak activation $[6,17,18,24,26,28-31,34,38]$, hip abductor strength $[3-5,16,25,29]$, Trendelenburg sign $[5,16]$ hip abductor torque $[23,33]$, trigger points $[16,27,35,36]$, cross sectional area [32, 37], or muscle thickness [39]. Studies conducted these measurements non-weight bearing $[3,4,6,25,27,30,32-37,39]$, dynamically weight bearing $[17,23,26,38]$ or statically weight bearing $[24,28,31]$. Three studies utilised nonweight bearing measurements while also using dynamic and static measurements while weight bearing $[5,16,29]$.

All studies included participants with either nonspecific or unidentified LBP (Table 1). The length of time

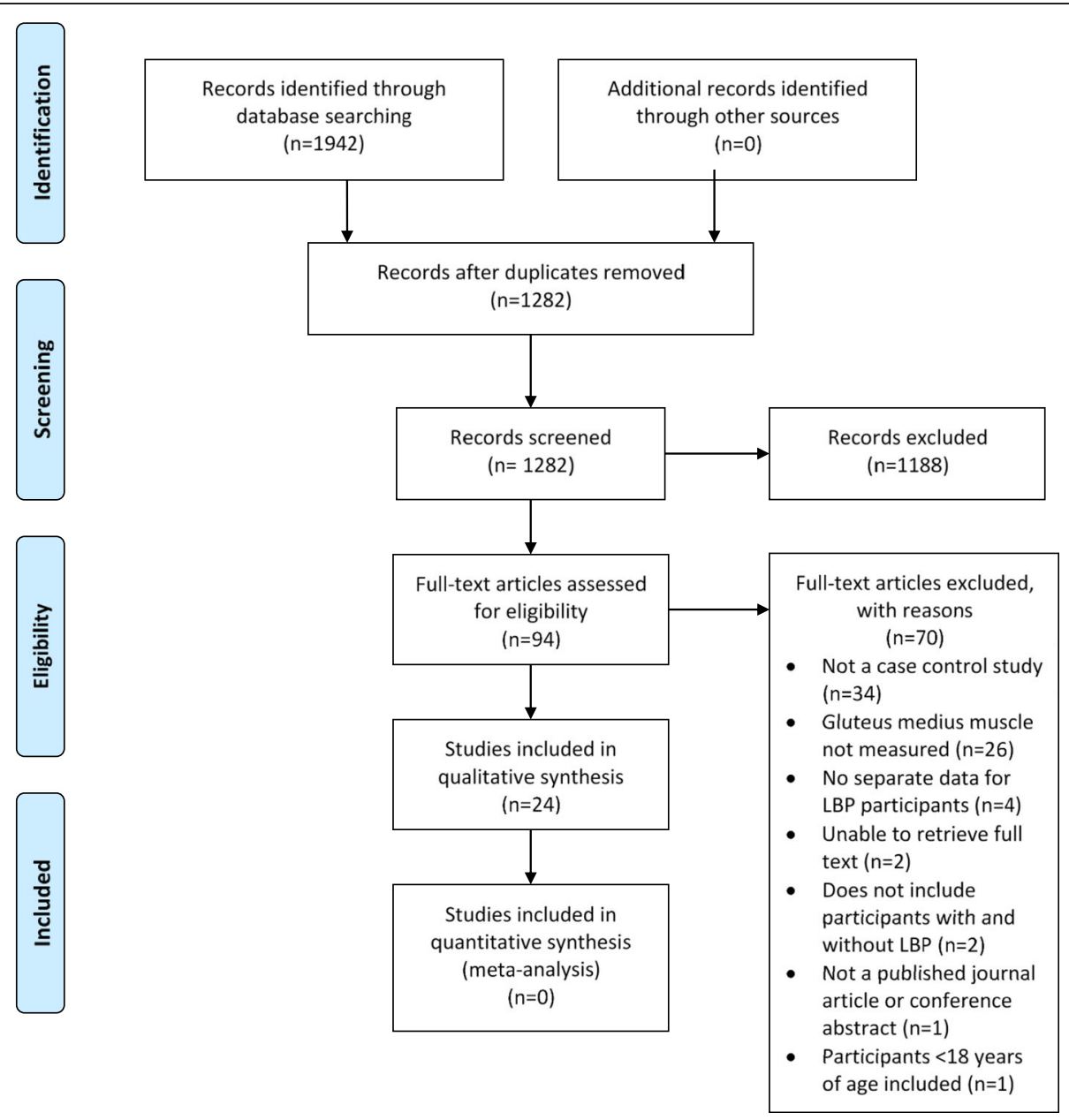

Fig. 1 PRIMSA flow diagram 
Table 1 Overview of included studies

\begin{tabular}{|c|c|c|c|c|c|c|}
\hline Study & Participants LBP Group & Participants control group & Type of LBP & Duration of LBP & $\begin{array}{l}\text { Presence of LBP at } \\
\text { Baseline }\end{array}$ & $\begin{array}{l}\text { Gluteus medius } \\
\text { measurement } \\
\text { outcome }\end{array}$ \\
\hline $\begin{array}{l}\text { Aboufazeli et al. } \\
2018 \text { [39] Iran. }\end{array}$ & $\begin{array}{l}n=30 \\
\text { Mean age, years (SD): } \\
34.6(6.2) \\
\text { Population: Not reported } \\
\text { Gender: } 100 \% \mathrm{~F} \\
\text { BMI (SD): } 23.4 \text { (3.2) }\end{array}$ & $\begin{array}{l}n=30 \\
\text { Mean age, years (SD): } \\
36.7 \text { (6.7) } \\
\text { Population: Note reported } \\
\text { Gender: } 100 \% \text { F } \\
\text { BMI (SD): } 23.6 \text { (3.3) }\end{array}$ & $\begin{array}{l}\text { Nonspecific } \\
\text { LBP }\end{array}$ & $\geq 3$ months & Yes & $\begin{array}{l}\text { Muscle } \\
\text { thickness }\end{array}$ \\
\hline $\begin{array}{l}\text { Arab \& } \\
\text { Nourbakhsh } 2010 \\
\text { [4] Iran }\end{array}$ & $\begin{array}{l}n=100 \\
\text { Mean age, years (SD): } \\
42.58 \text { (14.1) } \\
\text { Population: Patients of } \\
\text { orthopaedic department } \\
\text { Gender: Not Reported } \\
\text { BMI (SD): } 25.03 \text { (3.0) }\end{array}$ & $\begin{array}{l}n=100 \\
\text { Mean age, years (SD): } \\
43.40 \text { (4.41) } \\
\text { Population: Patients of } \\
\text { orthopaedic department } \\
\text { Gender: Not Reported } \\
\text { BMI (SD): } 25.68 \text { (4.1) }\end{array}$ & $\begin{array}{l}\text { Not } \\
\text { Reported }\end{array}$ & $>6$ weeks & Yes & Strength \\
\hline $\begin{array}{l}\text { Cai \& Kong } 2015 \\
\text { [23] Singapore }\end{array}$ & $\begin{array}{l}n=18 \\
\text { Mean age, years (SD): } \\
27.80 \text { (NR) } \\
\text { Population: Recreational } \\
\text { Runners } \\
\text { Gender: } 50 \% \mathrm{~F} \\
\text { BMI (SD): } 21.75 \text { (NR) }\end{array}$ & $\begin{array}{l}n=18 \\
\text { Mean age, years (SD): } \\
24.60 \text { (NR) } \\
\text { Population: Recreational } \\
\text { Runners } \\
\text { Gender: } 50 \% \text { F } \\
\text { BMI (SD): } 21.40 \text { (NR) }\end{array}$ & $\begin{array}{l}\text { Not } \\
\text { Reported }\end{array}$ & $\begin{array}{l}>3 \text { months, but } \\
\text { less than } 36 \\
\text { months }\end{array}$ & Yes & Strength \\
\hline $\begin{array}{l}\text { Cooper et al. } \\
2016 \text { [16] USA }\end{array}$ & $\begin{array}{l}n=150 \\
\text { Mean age, years (SD): } \\
41.40 \text { (13.0) } \\
\text { Population: Patients at } \\
\text { lowa Spine Centre } \\
\text { Physical Therapy Clinic } \\
\text { Gender: } 64.70 \% \mathrm{~F} \\
\text { BMI (SD): } 29.60 \text { (7.2) }\end{array}$ & $\begin{array}{l}n=75 \\
\text { Mean age, years (SD): } \\
40.70 \text { (13.9) } \\
\text { Population: Patients at } \\
\text { lowa Spine Centre Physical } \\
\text { Therapy Clinic } \\
\text { Gender: } 64.30 \% \text { F } \\
\text { BMI (SD): } 25.80 \text { (7.0) }\end{array}$ & $\begin{array}{l}\text { Nonspecific } \\
\text { LBP }\end{array}$ & $>3$ months & $\begin{array}{l}\text { Yes - Only one } \\
\text { participant reported } \\
\text { no current LBP }\end{array}$ & $\begin{array}{l}\text { Strength and } \\
\text { trigger points }\end{array}$ \\
\hline $\begin{array}{l}\text { Embaby \& } \\
\text { Abdallah } 2013 \\
\text { [24] Egypt }\end{array}$ & $\begin{array}{l}n=15 \\
\text { Mean age, years (SD): } \\
29.53 \text { (2.4) } \\
\text { Population Clinical } \\
\text { Instructors } \\
\text { Gender: } 100 \% \text { F } \\
\text { BMI (SD): } 24.01 \text { (NR) }\end{array}$ & $\begin{array}{l}n=15 \\
\text { Mean age, years (SD): } \\
29.07 \text { (2.4) } \\
\text { Population: Clinical } \\
\text { Instructors } \\
\text { Gender: } 100 \% \mathrm{~F} \\
\text { BMI (SD): } 22.63 \text { (NR) }\end{array}$ & Nonspecific & $\begin{array}{l}\text { Periods of mild or } \\
\text { moderate LBP for } \\
>6 \text { months }\end{array}$ & Not Reported & Fatigability \\
\hline $\begin{array}{l}\text { Farahpour et al. } \\
2018 \text { [17] Iran }\end{array}$ & $\begin{array}{l}n=15 \\
\text { Mean age, years (SD): } \\
25.30 \text { (2.9) } \\
\text { Population: Patients from } \\
\text { a 'clinic' } \\
\text { Gender:0\%F } \\
\text { BMI (SD): } 26.80(1.5)\end{array}$ & $\begin{array}{l}n=15 \\
\text { Mean age, years (SD): } \\
26.00(2.9) \\
\text { Population: Not reported } \\
\text { Gender: } 0 \% \mathrm{~F} \\
\text { BMI (SD): } 25.90(3.2)\end{array}$ & $\begin{array}{l}\text { Not } \\
\text { reported }\end{array}$ & Not reported & Yes & Activity level \\
\hline $\begin{array}{l}\text { Farasyn \& } \\
\text { Meeusen } 2005 \\
\text { [35] Belgium }\end{array}$ & $\begin{array}{l}n=87 \\
\text { Mean age, years (SD): } \\
43.00 \text { (13.0) } \\
\text { Population: Physiotherapy } \\
\text { patients } \\
\text { Gender: } 55.17 \% \mathrm{~F} \\
\text { BMI (SD): } 20.50(2.8)\end{array}$ & $\begin{array}{l}n=64 \\
\text { Mean age, years (SD): } \\
40.00 \text { (11.0) } \\
\text { Population: Physiotherapy } \\
\text { patients } \\
\text { Gender: } 62.50 \% \mathrm{~F} \\
\text { BMI (SD): } 21.50(3.2)\end{array}$ & $\begin{array}{l}\text { Nonspecific } \\
\text { LBP }\end{array}$ & $\begin{array}{l}\text { Subacute } \\
\text { nonspecific lower } \\
\text { back pain }\end{array}$ & Yes & Trigger points \\
\hline $\begin{array}{l}\text { Hides et al. } 2016 \\
\text { [25] Australia }\end{array}$ & $\begin{array}{l}\text { As a whole group } \\
\text { LBP } n=7 \text {, no } \operatorname{LBP} n=18 \\
\text { Mean age, years (SD): } \\
24.40 \text { (5.5) } \\
\text { Population: Elite } \\
\text { Footballers } \\
\text { Gender: } 0 \% \mathrm{~F} \\
\text { BMI (SD): } 23.61 \text { (NR) }\end{array}$ & & $\begin{array}{l}\text { Not } \\
\text { Reported }\end{array}$ & Not reported & Not reported & Strength \\
\hline $\begin{array}{l}\text { Hungerford et al. } \\
2003 \text { [26] } \\
\text { Australia }\end{array}$ & $\begin{array}{l}n=14 \\
\text { Mean age, years (SD): } \\
32.70 \text { (NR) } \\
\text { Population: Men with SIJP } \\
\text { Gender: } 0 \% \text { F }\end{array}$ & $\begin{array}{l}n=14 \\
\text { Mean age, years (SD): } \\
33.50 \text { (NR) } \\
\text { Population: without SIJP } \\
\text { Gender: 0\%F }\end{array}$ & SIJP & $>2$ months & Yes & $\begin{array}{l}\text { Activity level } \\
\text { and time to } \\
\text { onset }\end{array}$ \\
\hline
\end{tabular}


Table 1 Overview of included studies (Continued)

\begin{tabular}{|c|c|c|c|c|c|c|}
\hline Study & Participants LBP Group & Participants control group & Type of LBP & Duration of LBP & $\begin{array}{l}\text { Presence of LBP at } \\
\text { Baseline }\end{array}$ & $\begin{array}{l}\text { Gluteus medius } \\
\text { measurement } \\
\text { outcome }\end{array}$ \\
\hline & BMI (SD): 24.63 (NR) & BMI (SD):23.40 (NR) & & & & \\
\hline $\begin{array}{l}\text { Iglesias-Gonzalez } \\
\text { et al. } 2013 \text { [27] } \\
\text { Spain }\end{array}$ & $\begin{array}{l}n=42 \\
\text { Mean age, years (SD): } \\
45.00 \text { (10.0) } \\
\text { Population: Patients of a } \\
\text { private physical therapy } \\
\text { clinic } \\
\text { Gender: } 50.00 \% F \\
\text { BMI (SD): } 24.50 \text { (3.2) }\end{array}$ & $\begin{array}{l}n=42 \\
\text { Mean age, years (SD): } \\
45.00 \text { (9.0) } \\
\text { Population: Subjects who } \\
\text { responded to local } \\
\text { advertisements } \\
\text { Gender: } 50.00 \% \mathrm{~F} \\
\text { BMI (SD): } 24.90 \text { (3.4) }\end{array}$ & $\begin{array}{l}\text { Nonspecific } \\
\text { LBP }\end{array}$ & $>3$ years & Yes & Trigger points \\
\hline $\begin{array}{l}\text { Kendall et al. } \\
2010 \text { [5] Canada }\end{array}$ & $\begin{array}{l}n=10 \\
\text { Mean age, years (SD): } \\
32.00 \text { (NR) } \\
\text { Population: Not Stated } \\
\text { Gender: } 80.00 \% \text { F } \\
\text { BMI (SD): } 20.86 \text { (NR) }\end{array}$ & $\begin{array}{l}n=10 \\
\text { Mean age, years (SD): } \\
26.00 \text { (NR) } \\
\text { Population: Not Stated } \\
\text { Gender: } 80.00 \% \mathrm{~F} \\
\text { BMI (SD): } 21.61 \text { (NR) }\end{array}$ & $\begin{array}{l}\text { Nonspecific } \\
\text { LBP }\end{array}$ & $>6$ weeks & Yes & Strength \\
\hline $\begin{array}{l}\text { Larsen et al. } 2018 \\
\text { [38] Denmark }\end{array}$ & $\begin{array}{l}n=27 \\
\text { Mean age, years (SD): } \\
27.40 \text { (9.9) } \\
\text { Population: University } \\
\text { campus and hospital } \\
\text { Gender: } 44.44 \\
\text { BMI (SD): } 21.90 \text { (3.2) }\end{array}$ & $\begin{array}{l}n=26 \\
\text { Mean age, years (SD): } \\
23.60 \text { (4.4) } \\
\text { Population: University } \\
\text { campus and hospital } \\
\text { Gender: } 61.53 \% \text { F } \\
\text { BMI (SD): } 23.80(2.5)\end{array}$ & Nonspecific & $>3$ years & No & Activity level \\
\hline $\begin{array}{l}\text { Mendis et al. } \\
2016 \text { [37] } \\
\text { Australia }\end{array}$ & $\begin{array}{l}\text { As a whole group } \\
\text { LBP } n=13 \text {, no LBP } \\
n=33 \\
\text { Mean age, years (SD): } \\
22.80 \text { (3.5) } \\
\text { Population: Australian } \\
\text { Elite AFL Players } \\
\text { Gender: } 0 \% \text { F } \\
\text { BMI (SD): } 25.00 \text { (NR) }\end{array}$ & & $\begin{array}{l}\text { Not } \\
\text { Reported }\end{array}$ & Not Reported & Yes & $\begin{array}{l}\text { Cross-sectional } \\
\text { area }\end{array}$ \\
\hline $\begin{array}{l}\text { Nelson-Wong } \\
\text { et al. } 2013 \text { [6] } \\
\text { USA }\end{array}$ & $\begin{array}{l}n=17 \\
\text { Mean age, years (SD): } \\
27.71 \text { (10.6) } \\
\text { Population: general } \\
\text { population } \\
\text { Gender: not reported } \\
\text { BMI (SD): } 23.42 \text { (2.9) }\end{array}$ & $\begin{array}{l}n=17 \\
\text { Mean age, years (SD): } \\
28.52 \text { (10.2) } \\
\text { Population: general } \\
\text { population } \\
\text { Gender: not reported } \\
\text { BMI (SD): } 22.99(1.8)\end{array}$ & $\begin{array}{l}\text { Not } \\
\text { Reported }\end{array}$ & Not Reported & Yes & Time to onset \\
\hline $\begin{array}{l}\text { Njoo \& Van der } \\
\text { Does } 1994 \text { [36] } \\
\text { Netherlands }\end{array}$ & $\begin{array}{l}n=61 \\
\text { Mean age, years (SD): } \\
36.20(9.8) \\
\text { Population: Patients of } \\
\text { participating health care } \\
\text { centres } \\
\text { Gender: } 44.20 \% \text { F } \\
\text { BMI (SD): Not Reported }\end{array}$ & $\begin{array}{l}n=61 \\
\text { Mean age, years (SD): } \\
38.10 \text { (9.9) } \\
\text { Population: Every 10th } \\
\text { patients of health care } \\
\text { centres } \\
\text { without LBP } \\
\text { Gender: } 50.70 \% \text { F } \\
\text { BMI (SD): Not Reported }\end{array}$ & $\begin{array}{l}\text { Nonspecific } \\
\text { LBP }\end{array}$ & $\begin{array}{l}\text { Recent episode of } \\
\text { less than } 2 \text { months }\end{array}$ & No & Trigger points \\
\hline $\begin{array}{l}\text { Notzel et al. } 2011 \\
\text { [28] Germany }\end{array}$ & $\begin{array}{l}n=8 \\
\text { Mean age, years (SD): } \\
42.40 \text { (14.5) } \\
\text { Population: Not Reported } \\
\text { Gender: } 100 \% \mathrm{~F} \\
\text { BMI (SD): } 23.10(2.4)\end{array}$ & $\begin{array}{l}n=12 \\
\text { Mean age, years (SD): } \\
27.30 \text { (7.1) } \\
\text { Population: Not Reported } \\
\text { Gender: } 100 \% \mathrm{~F} \\
\text { BMI (SD): } 20.40(2.6)\end{array}$ & $\begin{array}{l}\text { Nonspecific } \\
\text { LBP }\end{array}$ & $\begin{array}{l}>6 \text { months several } \\
\text { times per week or } \\
\text { daily }\end{array}$ & Not Reported & Activity level \\
\hline $\begin{array}{l}\text { Nourbakhsh \& } \\
\text { Arab } 2002 \text { [3] Iran }\end{array}$ & $\begin{array}{l}n=300 \\
\text { Mean age, years (SD): } \\
43.00 \text { (NR) } \\
\text { Population: Hospital } \\
\text { inpatients } \\
\text { Gender: } 50.00 \% \mathrm{~F} \\
\text { BMI (SD): } 25.76 \text { (NR) }\end{array}$ & $\begin{array}{l}n=300 \\
\text { Mean age, years (SD): } \\
43.00 \text { (NR) } \\
\text { Population: Hospital } \\
\text { inpatients } \\
\text { Gender: } 50.00 \% \mathrm{~F} \\
\text { BMl (SD): } 24.44 \text { (NR) }\end{array}$ & $\begin{array}{l}\text { Not } \\
\text { Reported }\end{array}$ & $>6$ weeks & Yes & Strength \\
\hline Penney et al. & $n=21$ & $n=22$ & Nonspecific & $>12$ weeks & Yes & Activity level, \\
\hline
\end{tabular}


Table 1 Overview of included studies (Continued)

\begin{tabular}{|c|c|c|c|c|c|c|}
\hline Study & Participants LBP Group & Participants control group & Type of LBP & Duration of LBP & $\begin{array}{l}\text { Presence of LBP at } \\
\text { Baseline }\end{array}$ & $\begin{array}{l}\text { Gluteus medius } \\
\text { measurement } \\
\text { outcome }\end{array}$ \\
\hline 2014 [29] Canada & $\begin{array}{l}\text { Mean age, years (SD): } \\
46.00 \text { (15.2) } \\
\text { Population: Patients at } \\
\text { local physiotherapy clinics } \\
\text { Gender: } 42.85 \% \mathrm{~F} \\
\text { BMI (SD): } 27.40 \text { (NR) }\end{array}$ & $\begin{array}{l}\text { Mean age, years (SD): } \\
44.00 \text { (15.5) } \\
\text { Population: University and } \\
\text { Hospital Community } \\
\text { Gender: } 33.36 \% \mathrm{~F} \\
\text { BMI (SD): } 26.95 \text { (NR) }\end{array}$ & LBP & & & $\begin{array}{l}\text { time to onset, } \\
\text { and strength }\end{array}$ \\
\hline $\begin{array}{l}\text { Rabel et al. } 2013 \\
\text { [30] USA }\end{array}$ & $\begin{array}{l}n=12 \\
\text { Mean age, years (SD): } \\
44.40 \text { (14.6) } \\
\text { Population: Physiotherapy } \\
\text { patients } \\
\text { Gender: } 58.33 \% \mathrm{~F} \\
\text { BMI (SD): } 29.70 \text { (10.3) }\end{array}$ & $\begin{array}{l}n=22 \\
\text { Mean age, years (SD): } \\
27.20 \text { (4.6) } \\
\text { Population: Recreationally } \\
\text { active } \\
\text { Gender: } 50.00 \% \mathrm{~F} \\
\text { BMI (SD): } 24.70(4.9)\end{array}$ & $\begin{array}{l}\text { Not } \\
\text { Reported }\end{array}$ & $\begin{array}{l}<2 \text { months and } \\
>3 / 10 \text { on VAS }\end{array}$ & Yes & Time to onset \\
\hline $\begin{array}{l}\text { Ringheim et al. } \\
2015 \text { [31] Norway }\end{array}$ & $\begin{array}{l}n=17 \\
\text { Mean age, years (SD):39.00 } \\
\text { (5.4) } \\
\text { Population: Hospital } \\
\text { outpatients } \\
\text { Gender: } 58.82 \% \mathrm{~F} \\
\text { BMI (SD): } 25.90(4.7)\end{array}$ & $\begin{array}{l}n=20 \\
\text { Mean age, years (SD): } \\
40.20 \text { (5.4) } \\
\text { Population: Not Reported } \\
\text { Gender: } 61.90 \% \text { F } \\
\text { BMI (SD): } 25.20 \text { (3.7) }\end{array}$ & Nonspecific & $>3$ months & Yes & $\begin{array}{l}\text { Activity level } \\
\text { and fatigability }\end{array}$ \\
\hline $\begin{array}{l}\text { Santos et al. } 2013 \\
\text { [18] Brazil }\end{array}$ & $\begin{array}{l}n=29 \\
\text { Mean age, years (SD): } \\
45.80 \text { (14.3) } \\
\text { Population: Orthopaedic } \\
\text { patients } \\
\text { Gender: } 100 \% F \\
\text { BMI (SD): } 24.15 \text { (3.9) }\end{array}$ & $\begin{array}{l}n=30 \\
\text { Mean age, years (SD): } \\
\text { 44.57 (13.6) } \\
\text { Population: Not Reported } \\
\text { Gender: } 100 \% \mathrm{~F} \\
\text { BMI (SD): } 23.77 \text { (2.1) }\end{array}$ & $\begin{array}{l}\text { Nonspecific } \\
\text { LBP }\end{array}$ & $>3$ months & No & $\begin{array}{l}\text { Activity level } \\
\text { and time of } \\
\text { peak }\end{array}$ \\
\hline $\begin{array}{l}\text { Skorupska et al. } \\
2016 \text { [32] Poland }\end{array}$ & $\begin{array}{l}n=71 \\
\text { Mean age, years (SD): } \\
47.70 \text { (8.4) } \\
\text { Population: Not reported } \\
\text { Gender: } 61.97 \% F \\
\text { BMI (SD): Not Reported }\end{array}$ & $\begin{array}{l}n=29 \\
\text { Mean age, years (SD): } \\
47.60 \text { (9.9) } \\
\text { Population: Not reported } \\
\text { Gender: } 65.51 \% F \\
\text { BMI (SD): Not Reported }\end{array}$ & $\begin{array}{l}\text { Not } \\
\text { Reported }\end{array}$ & $\begin{array}{l}\text { Subacute or } \\
\text { Chronic }\end{array}$ & Yes & $\begin{array}{l}\text { Cross-sectional } \\
\text { area }\end{array}$ \\
\hline $\begin{array}{l}\text { Sutherlin \& Hart } \\
\text { 2015a [33] USA }\end{array}$ & $\begin{array}{l}n=12 \\
\text { Mean age, years (SD): } \\
24.00 \text { (4.0) } \\
\text { Population: Not Reported } \\
\text { Gender: Not Reported \%F } \\
\text { BMI (SD): } 25.19 \text { (3.4) }\end{array}$ & $\begin{array}{l}n=12 \\
\text { Mean age, years (SD): } \\
22.00 \text { (3.0) } \\
\text { Population: Not } \\
\text { Reported } \\
\text { Gender: Not Reported \%F } \\
\text { BMI (SD): } 21.28 \text { (2.4) }\end{array}$ & $\begin{array}{l}\text { Not } \\
\text { Reported }\end{array}$ & Not reported & Yes & Strength \\
\hline $\begin{array}{l}\text { Sutherlin \& Hart } \\
\text { 2015b [34] USA }\end{array}$ & $\begin{array}{l}n=12 \\
\text { Mean age, years (SD): } \\
24.00 \text { (4.0) } \\
\text { Population: Not Reported } \\
\text { Gender: } 58.33 \% \text { F } \\
\text { BMI (SD): } 25.19 \text { (3.4) }\end{array}$ & $\begin{array}{l}n=12 \\
\text { Mean age, years (SD): } \\
22.0 \text { (3.0) } \\
\text { Population: Not } \\
\text { Reported } \\
\text { Gender: } 75.00 \% \text { F } \\
\text { BMI (SD): } 21.28 \text { (2.4) }\end{array}$ & $\begin{array}{l}\text { Not } \\
\text { Reported }\end{array}$ & Not reported & Yes & $\begin{array}{l}\text { Activity level } \\
\text { and fatigability }\end{array}$ \\
\hline
\end{tabular}

SD standard deviation, BMI body mass index, LBP low back pain, NR not reported, SLR single leg raise, T12 12th thoracic vertebra, SIJP Sacroiliac joint pain, SIJ Sacroiliac joint

participants had LBP within each of the studies varied. Some included acute [25], subacute [4, 35], chronic $[16,18,23,24,27-29,31,38,39]$, or mixed/unclear durations of LBP [3, 5, 6, 17, 26, 30, 32-34, 36, 37].

Most studies performed generally well on the quality appraisal tool (Additional file 2). However, in the majority of studies there was insufficient information to determine how controls were recruited and there were inconsistencies in the type and number of potential confounding factors that were addressed.

\section{Included studies by measurement outcome Level of muscle activity (EMG)}

Eight studies $[17,18,26,28,29,31,34,38]$ measured the amount of activity in the gluteus medius muscle with EMG (Table 2). Of these studies, five [18, 26, 28, 29, 31] 


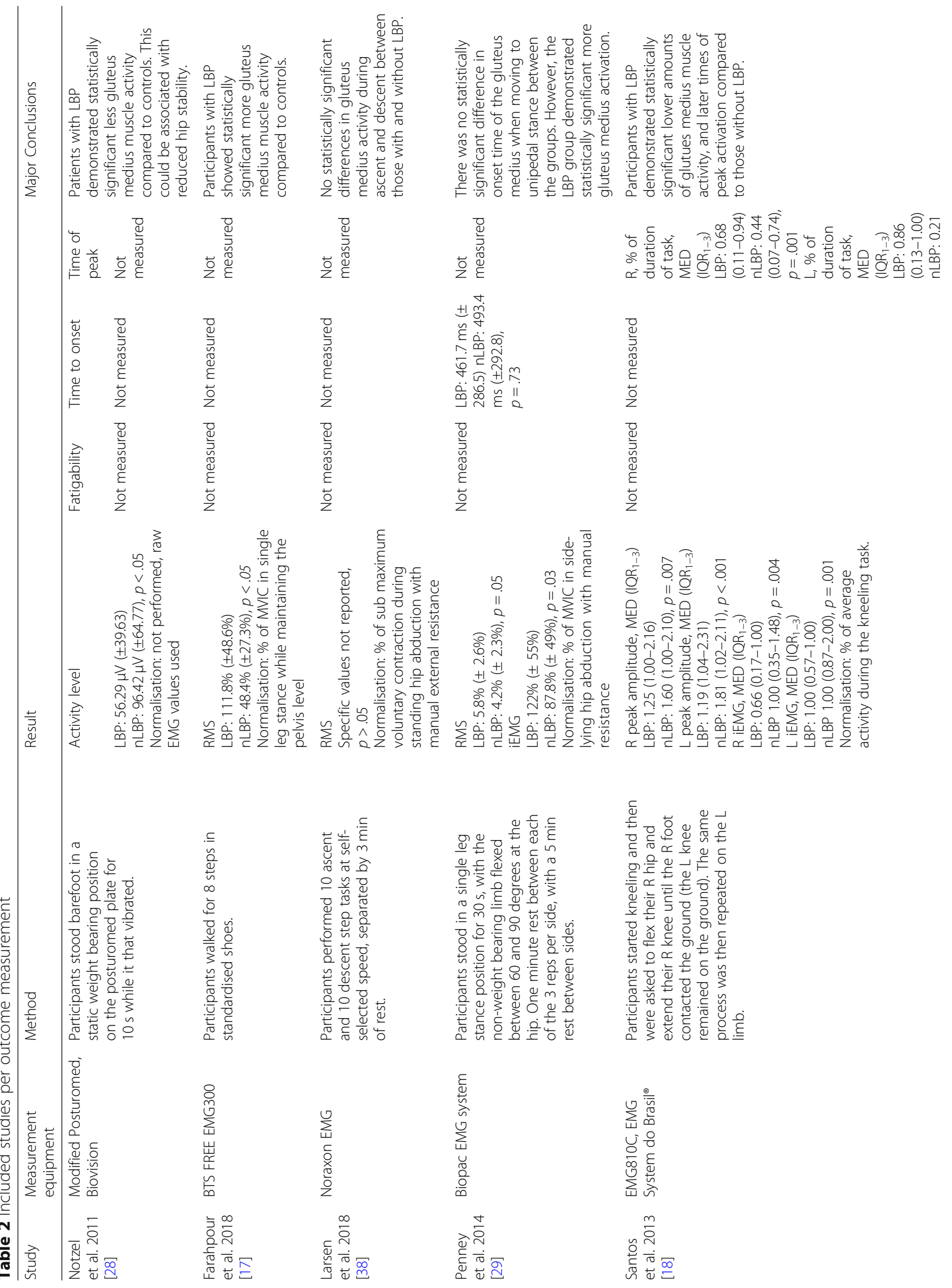




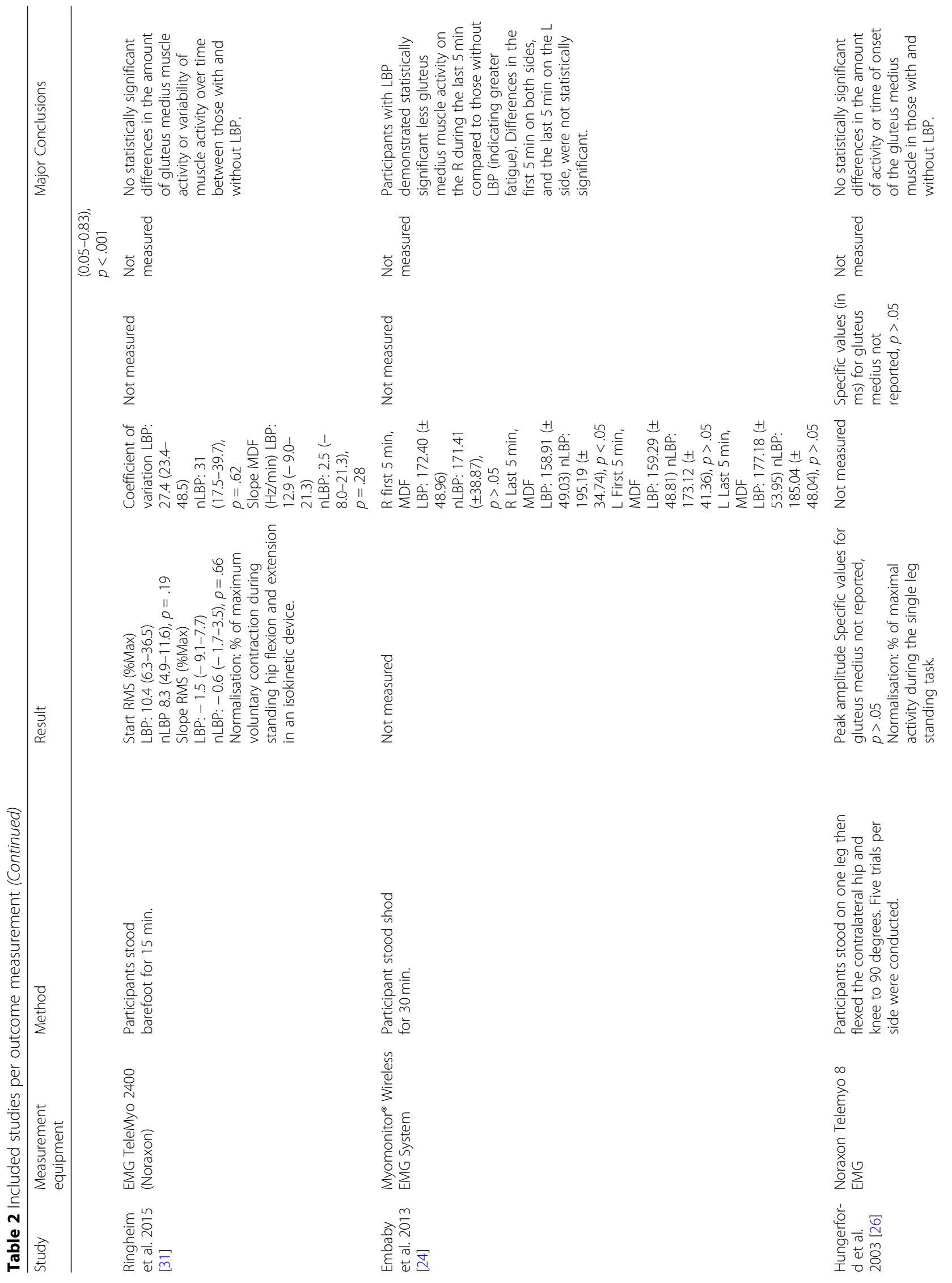




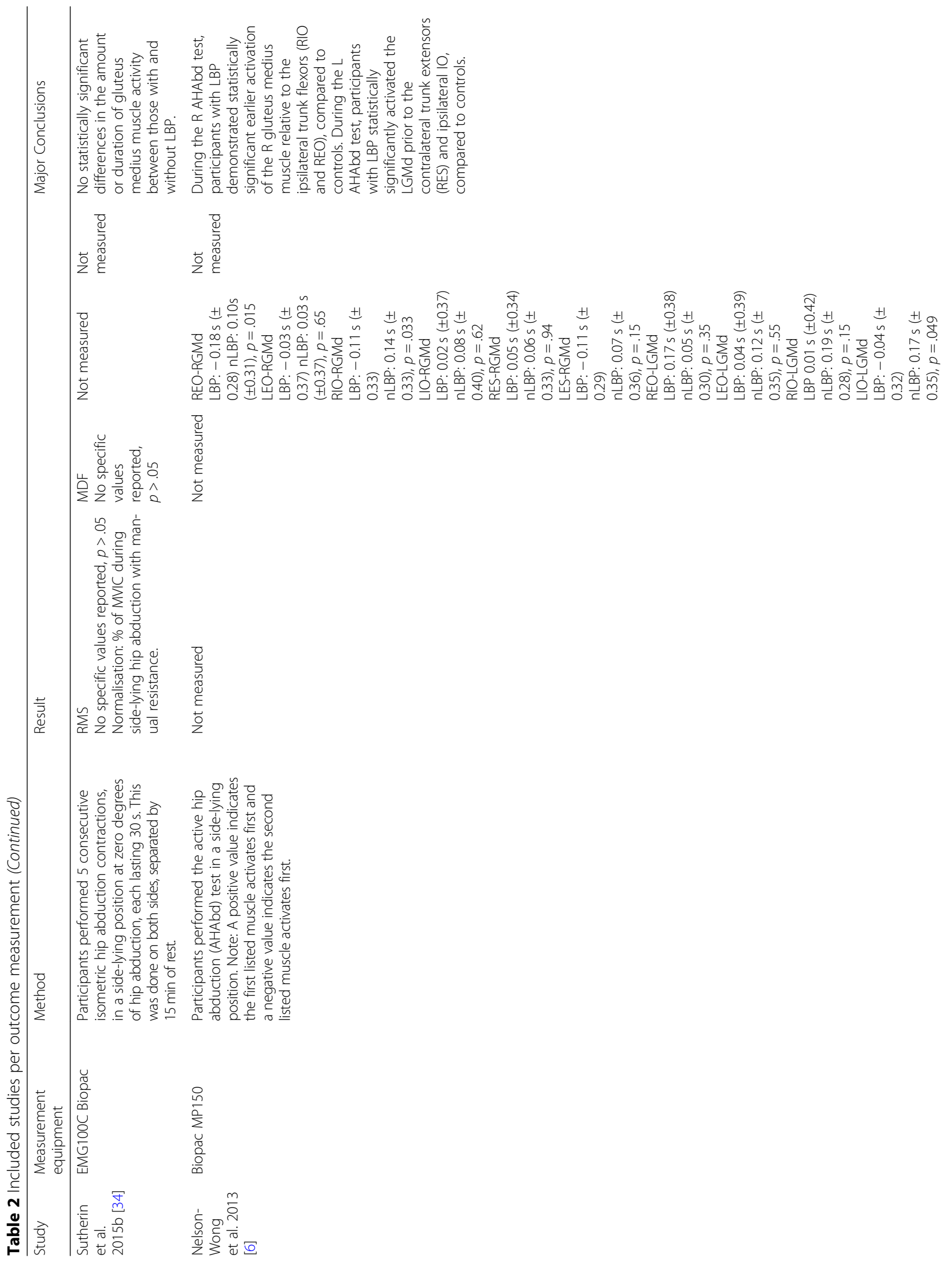




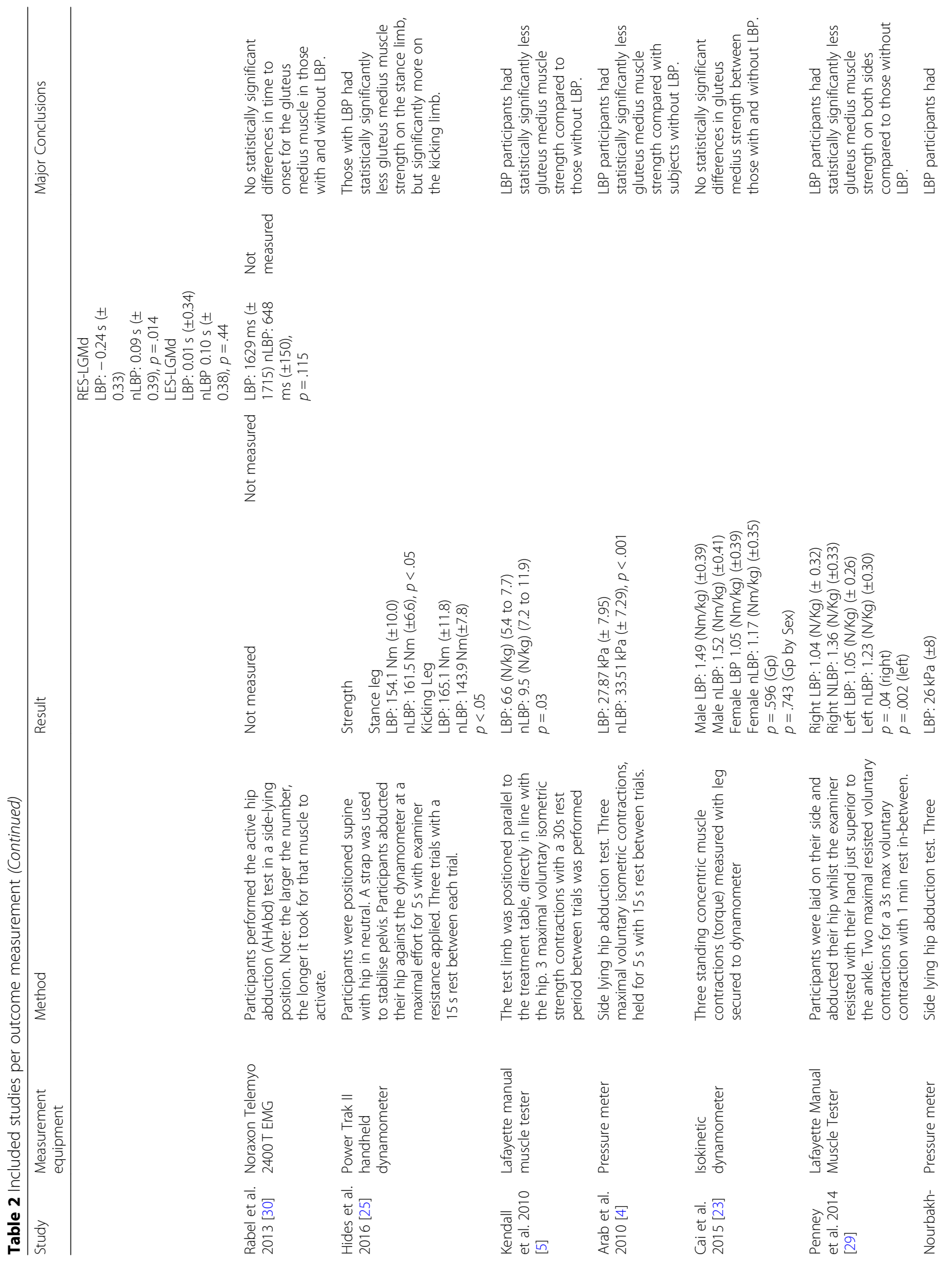




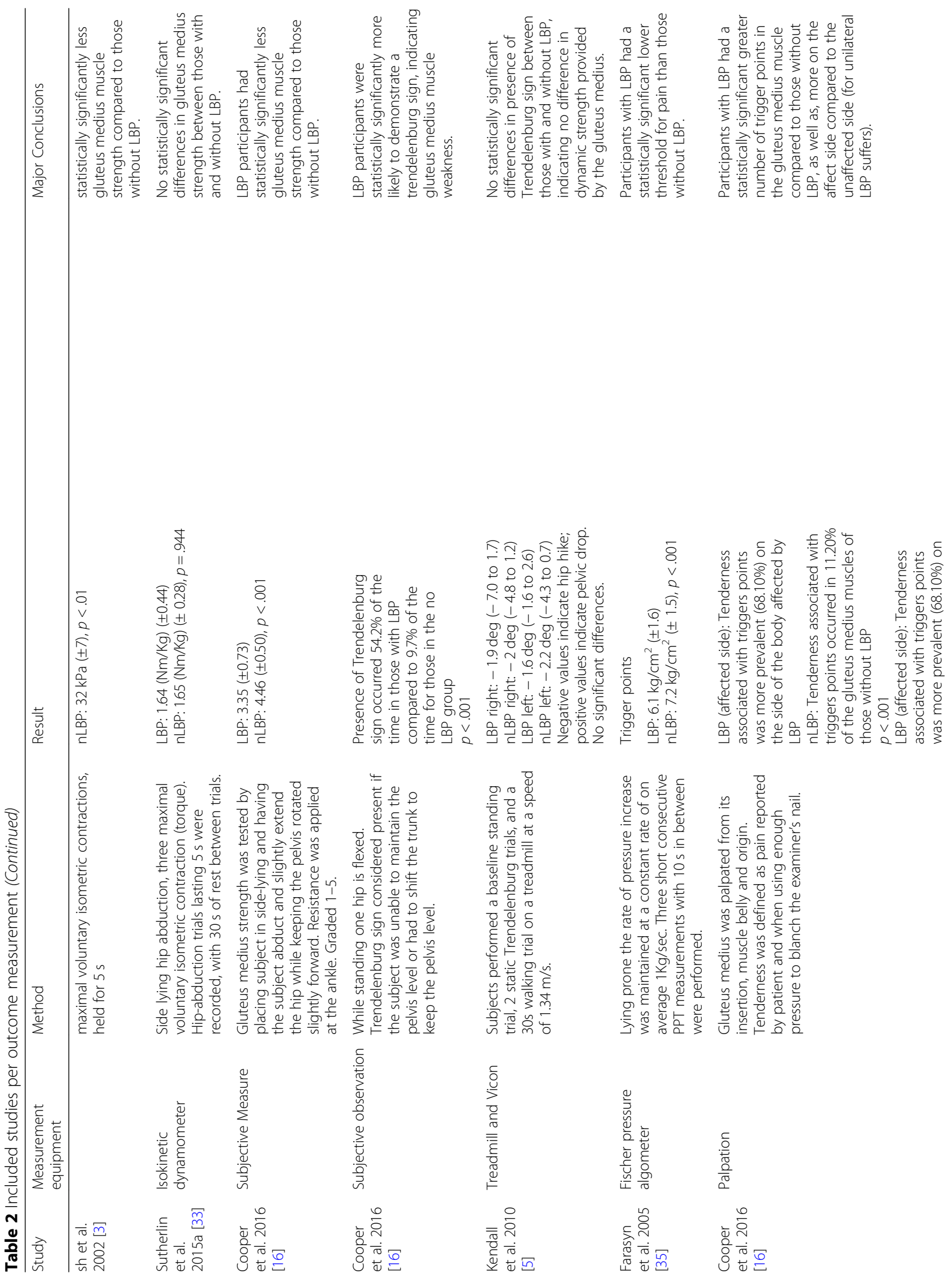




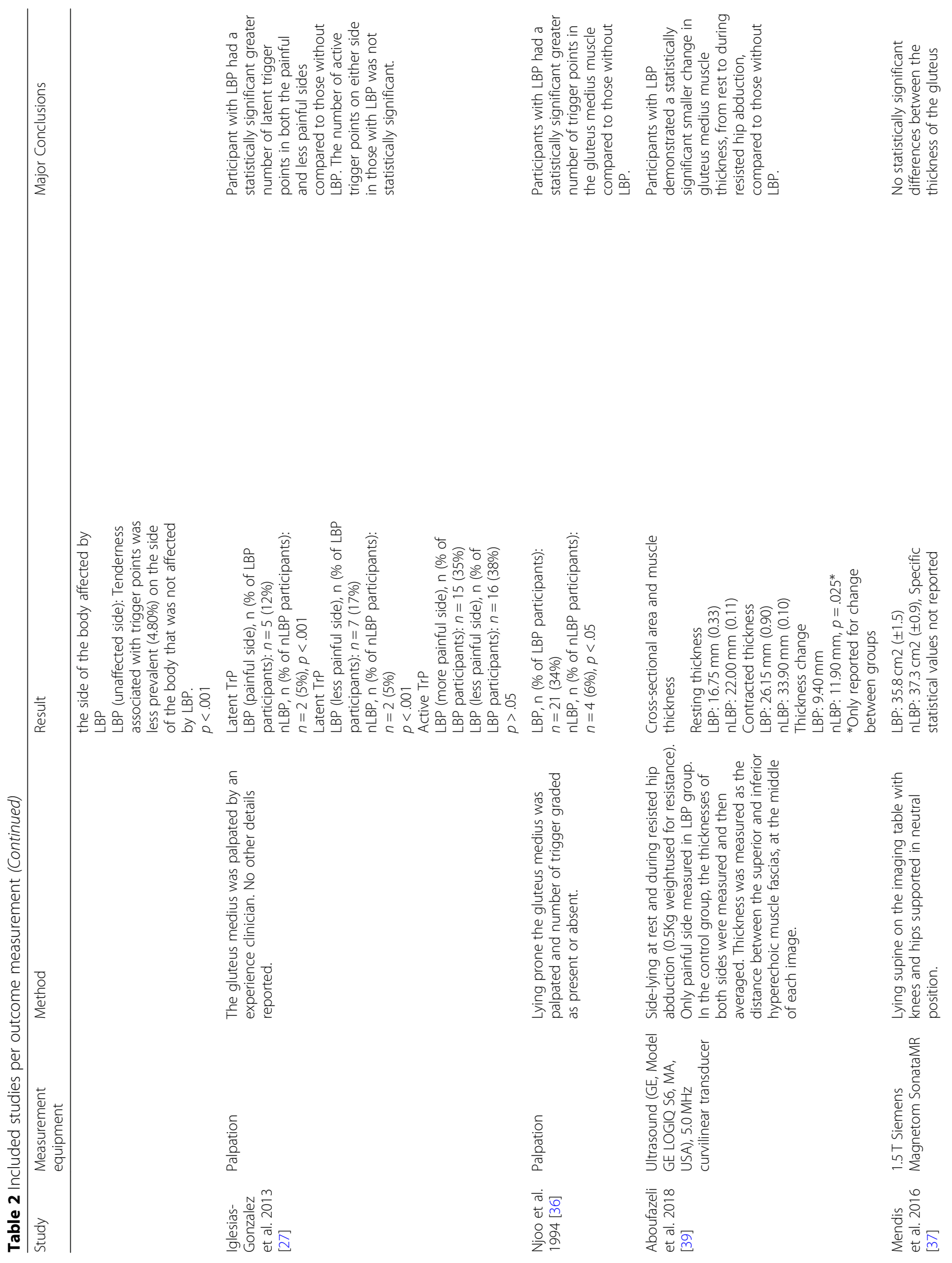




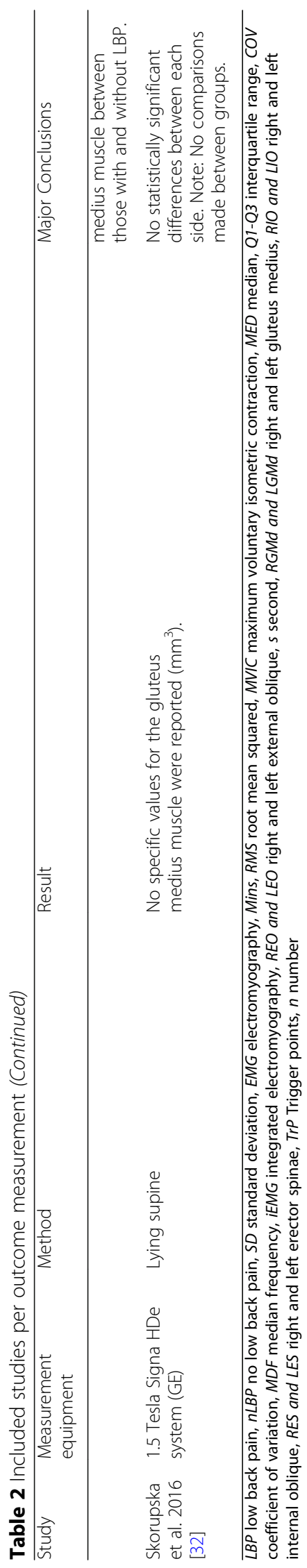


measured gluteus medius muscle activity in a static weight bearing position, with mixed results. Two studies found less gluteus medius muscle activity in those with LBP [18, 28], one study found more activity in those with LBP [29], and the remaining two studies found no difference in gluteus medius muscle activity between those with and without LBP [26, 31]. A further two studies measured gluteus medius muscle activity dynamically, with one study finding less gluteus medius muscle activity in those with LBP [17], and the other study finding no difference between those with and without LBP [38]. The remaining study measured gluteus medius muscle activity in a non-weight bearing position and found no difference in gluteus medius muscle activity between those with and without LBP [34].

\section{Fatigability (EMG)}

Three studies [24, 31, 34] measured the fatigability of the gluteus medius muscle with EMG (Table 2). Embaby et al. [24] found that those with LBP demonstrated statistically significant greater gluteus medius muscle fatigability after $30 \mathrm{~min}$ of standing compared to those without LBP, although this finding was only on the right side. Of the other two studies, one measured gluteus medius fatigability in a static weight bearing position [31], and the other in a non-weight bearing position [34], with both finding no statistically significant differences in the rate of gluteus medius muscle fatigability between those with and without LBP.

\section{Time to onset (EMG)}

Four studies [12, 26, 29, 30] measured the time it took for the gluteus medius muscle to activate with EMG (Table 2). Of these studies, two measured time to activation in a static single leg weight bearing position, with one requiring participants to abduct their ipsilateral hip [29], and the other study requiring participants to flex the contralateral hip [26]. Both studies found no statistically significant differences between those with and without LBP. The remaining two studies measured the time it took the gluteus medius muscle to activate during the non-weight bearing active hip abduction test [12, 30]. Nelson-Wong et al. [12] found that participants with LBP demonstrated statistically significant earlier activation of the gluteus medius, compared to some other trunk muscles (Table 2). This was in contrast to Rabel et al. [30] who found no statistically significant differences in time to activation of the gluteus medius muscle between those with and without LBP.

\section{Time to peak (EMG)}

One study [18] measured time to peak gluteus medius muscle activity during a static non-weight bearing kneeling task (Table 2). Participants with LBP took a statistically significant longer amount of time to reach peak activation compared to those without LBP.

\section{Strength}

Eight studies $[3-5,16,23,25,29,33]$ measured the strength of the gluteus medius muscle, with two of these studies $[5,16]$ measuring strength in more than one way (Table 2). Of these studies, seven measured gluteus medius muscle strength in a non-weight bearing side-lying hip abduction test with participants instructed to perform maximal effort against assessor $[3-5,16,29]$ or machine applied resistance $[25,33]$. The majority of the studies demonstrated a statistically significant reduction in gluteus medius muscle strength in those with LBP compared to those without LBP $[3-5,16,25,29]$, with the remaining study finding no difference [33].

Two studies [16, 23] measured gluteus medius muscle strength in a static weight bearing position (Table 2). One study measured concentric strength of the gluteus medius muscle in a standing position with a dynamometer and found no difference in strength between those with and without LBP [23]. Cooper et al. [16] measured gluteus medius strength statically using the static Trendelenburg test and found that those with LBP demonstrated a positive sign more often that those without LBP $(p<.001)$, indicating reduced gluteus medius muscle strength.

One study [5] measured gluteus medius muscle strength during gait using the Trendelenburg sign and found no statically significant differences between those with and without LBP.

\section{Trigger points in the gluteus medius muscle}

Four studies [16, 27, 35, 36] investigated gluteus medius trigger points (Table 2). Of these studies, three [16, 27, 36] used manual palpation and found that those with LBP had statistically significant greater number of trigger points in the gluteus medius compared to those without LBP. The remaining study used an algometry device to measure pressure pain thresholds as a representation of areas of tenderness in the gluteus medius [35]. They found that the threshold of pressure tolerance was lower in the LBP participants compared to those without LBP $(p<.001)$.

\section{Cross sectional area and muscle thickness}

One study [39] used ultrasound to investigate the change in thickness of the gluteus medius muscle between a resting state and during resisted hip abduction (Table 2). The authors found that those with LBP demonstrated a statistically significant smaller change in muscle thickness, from rest to during resisted hip abduction, compared to those without LBP $(p=.025)$. 
The other two studies investigated the cross sectional muscle area of the gluteus medius muscle using magnetic resonance imaging [32, 37] (Table 2). Both studies compared side to side differences within individual participants, due to participants having unilateral LBP [32], or LBP and no LBP participants being grouped together [37], with no significant differences found.

\section{Discussion}

This systematic review included 24 case-control studies investigating gluteus medius function in people with and without LBP. The findings for gluteus medius muscle activity $[17,18,26,28,29,31,34,38]$, fatigability [24, 31, 34], time to onset $[12,26,29,30]$, and time to peak activation [18] were mixed. Five of the eight studies measuring gluteus medius muscle strength demonstrated it to be significantly lower in those with LBP compared to those without LBP $[3,4,16,25,29]$. However, two of the eight studies found no difference [23,33], and the final study had mixed findings of significantly less gluteus medius muscle strength during side-lying hip abduction in those with LBP compared to those without LBP, but no differences in strength of this muscle between these groups during the Trendelenburg test [5]. Additionally, four studies investigating the presence of trigger points $[16,27,36]$ or areas of tenderness associated with trigger points [35], consistently showed that people with LBP are more likely to have higher numbers of trigger points and greater levels of tenderness in the gluteus medius muscle compared to those without LBP. The two studies that measured gluteus medius crosssectional area found no differences [32, 37], however, another study that measured gluteus medius muscle thickness using ultrasound found that those with LBP had a significantly smaller increase in gluteus medius thickness during side-lying hip abduction [39]. Due to differences in measurement techniques (Table 2), and the type and duration of LBP (Table 1), combining studies in a meta-analysis was not possible.

The majority (9 out of 11) of studies using EMG to assess gluteus medius muscle function did so in either non-weight bearing $[12,30,34]$ or static weight bearing tasks $[18,24,26,28,29,31]$. Generally, only a small range of EMG variables were reported within individual studies and variables were not consistent across multiple studies. Further investigation of possible differences in dynamic gluteus medius function in those with and without LBP, and whether dynamic function is predictive of LBP development, is required to help improve our understanding of the role of this muscle in the presence and development of LBP. Inclusion of other EMG outcome variables, such as mean amplitude, minimum level of activity, or change from minimum to maximum amplitude may provide additional insight into how this muscle functions.
The reduction in gluteus medius strength reported by the majority of studies is consistent with previously reported theoretical links between biomechanical dysfunction of the lumbopelvic-hip complex and lower limb, and the development of LBP [40]. During normal gait, the gluteus medius is responsible for producing and controlling transverse plane rotation and frontal plane position of the hip joint [41]. It is proposed that weakness of the gluteus medius results in several biomechanical changes that alter the position and stability of the pelvis and may subsequently contribute to LBP [40]. In the frontal plane, gluteus medius abduction weakness, which can be seen clinically as a positive Trendelenburg sign [42], is implicated in the development of a Trendelenburg gait pattern, with the pelvis dropping to the unsupported side during single leg weight bearing in the stance phase of gait [43]. This is suggested to cause uneven distribution of pressure on intervertebral discs and subsequent loading in the lumbar joints and so contribute to the development of LBP $[10,11]$. Similarly, reduced transverse plane control of the hip due to gluteus medius weakness is suggested to increase femoral adduction, internal femoral rotation and knee valgus $[44,45]$, causing anterior rotation of the ipsilateral pelvis, and altered lumbar spine loading, increasing the risk of LBP [46].

The consistent finding of increased numbers of active gluteus medius trigger points, as well as latent trigger points, in those with LBP $[16,27,36]$ may, in part, contribute to the gluteus medius dysfunction seen in this population. Recent evidence suggests that normal patterns of motor recruitment and movement efficiency can be affected by latent trigger points [47]. Additionally, there was a positive association between the mean number of active trigger points and the mean intensity of pain episodes. This suggests that the more trigger points that are present, the greater the severity of pain and likelihood of disruption to muscle activity patterns [27].

The secondary aim of this review was to investigate differences in gluteus medius function between types and durations of LBP. However, this was hampered by inconsistent definitions of LBP and the lack of detail of LBP type and duration reported in studies. Further differences between studies, such as the method for diagnosing LBP, the tool used to assess the severity of LBP, assessment techniques, and whether or not LBP participants had pain present at the time of assessment (Table 1) are additional areas that future research should attempt to standardise so that studies can be pooled in statistical analyses [48].

\section{Limitations}

This review was designed to be robust and comprehensive however it is possible that not all studies were identified. The likelihood of this occurring was reduced by a robust 
search strategy and independent title and abstract screening by two researchers. The generalisability of the review's findings also needs to be considered. Only studies that measured gluteus medius function in participants over the age of 18 were included. This coupled with the small number of studies per measurement outcome, differences in study methodology and population, and the unclear or inconsistent definitions of the type and duration of LBP has precluded more sophisticated methods of analysis. These differences may, in part, explain some of the insignificant findings between cases and controls within studies and could also have diluted the findings of this review, perhaps explaining why our findings are unclear for some outcome measures. In addition, differing reliability of measures used may have affected the outcomes of the included studies. Although it was not the purpose of this review to determine measurement reliability, only ten of the included studies reported measurement reliability, with large variability between studies for the same measurement outcome [3, 16, 25, 26, 30, 32, 35-37, 39]. Poor reliability of any measurement can account for insignificant findings where differences between cases and controls are small. This may be relevant to the results of studies included in this review and we suggest a comprehensive investigation of the existing reliability in this area be undertaken. The findings of this systematic review should be interpreted with caution and in context of the limitations of the review itself and those of the individual studies. Nevertheless, this systematic review provides a summary of the available literature which can be used to inform both clinical practice and future research.

\section{Conclusion}

In summary, we found that in those with LBP the gluteus medius muscle had reduced strength and more trigger points compared to those without LBP. Findings for the level of muscle activity, fatigability, time to onset, time to peak amplitude, cross sectional area, and muscle thickness were mixed. When interpreting these findings in context of the management of LBP patients, significant caution is recommended because the aim of this review was not to investigate intervention effectiveness. However, strengthening the gluteus medius muscle and eliminating trigger points may form an important part of the multidisciplinary management of LBP patients, although further research is needed before this can be confidently recommended. To help reduce inconsistencies in future research, the authors recommended following the standardised eligibility criteria outlined by Amundsen et al. [48]. Additionally, future research should aim to prospectively assess gluteus medius muscle function, with static and dynamic tasks across a range of outcome measures, and in those with and without LBP.

\section{Supplementary information}

Supplementary information accompanies this paper at https://doi.org/10. 1186/s12891-019-2833-4

Additional file 1. Key word search.

Additional file 2. Quality appraisal.

Additional file 3. Table of excluded full text articles.

Abbreviations

EMG: Electromyography; LBP: Low back pain

Acknowledgements

Not applicable.

Ethics approval and consent for publication

Not applicable.

Authors' contributions

SS, SC, and VC contributed to the conception and design of the review; analysis and interpretation of data; and drafting and revising of the manuscript. SC and BP performed data extraction and quality appraisal. MS contributed to the analysis and interpretation of data, and revising the manuscript. All authors have read and approved the final manuscript.

\section{Funding}

No funding was obtained for this study.

Availability of data and materials

All of the data for this study are contained in the manuscript, the additional files, or the individual studies included in this systematic review.

Consent for publication

Not applicable.

Competing interests

The authors declare that they have no competing interests.

\section{Author details}

'Discipline of Podiatry, University of Newcastle, Ourimbah, NSW 2258, Australia. ${ }^{2}$ Priority Research Centre for Physical Activity and Nutrition, University of Newcastle, Newcastle, Australia.

Received: 4 July 2019 Accepted: 13 September 2019

Published online: 22 October 2019

\section{References}

1. Hoy D, March L, Brooks P, Blyth F, Woolf A, Bain C, Williams G, Smith E, Vos T, Barendregt J, et al. The global burden of low back pain: estimates from the global burden of disease 2010 study. Ann Rheum Dis. 2014; 73(6):968-74.

2. Meucci R, Fassa A, Faria N. Prevalence of chronic low back pain: systematic review. Rev Saude Publica. 2015;49:1-1.

3. Nourbakhsh M, Arab A. Relationship between mechanical factors and incidence of low back pain. J Orthop Sports Phys Ther. 2002;32(9):447-60.

4. Arab A, Nourbakhsh $M$. The relationship between hip abductor muscle strength and iliotibial band tightness in individuals with low back pain. Chiropr Osteopat. 2010:18:1.

5. Kendall K, Schmidt C, Ferber R. The relationship between hip-abductor strength and the magnitude of pelvic drop in patients with low back pain. $J$ Sport Rehabil. 2010;19(4):422-35.

6. Nelson-Wong E, Poupore K, Ingvalson S, Dehmer K, Piatte A, Alexander S, Gallant P, McClenahan B, Davis AM. Neuromuscular strategies for lumbopelvic control during frontal and sagittal plane movement challenges differ between people with and without low back pain. J Electromyogr Kinesiol. 2013;23(6):1317-24.

7. Kanchanomai S, Janwantanakul P, Pensri P, Jiamjarasrangsi W. A prospective study of incidence and risk factors for the onset and persistence of low back pain in Thai university students. Asia Pac J Public Health. 2015:27(2): Np106-15. 
8. Lee D. An approach to examinations and treatment of lumbo-pelvic-hip region. 2nd ed. New York: Churchill Livingstone; 1999.

9. Fredericson M, Cookingham C, Chaudhari A, Dowdell B, Oestreicher N, Sahrmann S. Hip abductor weakness in distance runners with iliotibial band syndrome. Clin J Sport Med. 2000;10(3):169-75.

10. Gillan M, Ross J, McLean I, Porter R. The natural history of trunk list, its associated disability and the influence of McKenzie management. Eur Spine J. 1998; (6):480-3.

11. Dempsey A, Lloyd D, Elliott B, Steele J, Munro B. Changing sidestep cutting technique reduces knee valgus loading. Am J Sports Med. 2009;37(11): 2194-200.

12. Nelson-Wong E, Gregory D, Winter D, Callaghan J. Gluteus medius muscle activation patterns as a predictor of low back pain during standing. Clin Biomech. 2008;23(5):545-53.

13. Bussey MD, Kennedy JE, Kennedy G. Gluteus medius coactivation response in field hockey players with and without low back pain. Phys Ther Sport. 2016;17:24-9.

14. Marshall PW, Patel H, Callaghan JP. Gluteus medius strength, endurance, and co-activation in the development of low back pain during prolonged standing. Hum Mov Sci. 2011;30(1):63-73.

15. Nelson-Wong E, Callaghan JP. Is muscle co-activation a predisposing factor for low back pain development during standing? A multifactorial approach for early identification of at-risk individuals. J Electromyogr Kinesiol. 2010; 20(2):256-63.

16. Cooper N, Scavo K, Strickland K, Tipayamongkol N, Nicholson J, Bewyer D, Sluka K, Cooper NA, Scavo KM, Strickland K, et al. Prevalence of gluteus medius weakness in people with chronic low back pain compared to healthy controls. Eur Spine J. 2016;25(4):1258-65.

17. Farahpour $\mathrm{N}$, Jafarnezhadgero A, Allard P, Majlesi M. Muscle activity and kinetics of lower limbs during walking in pronated feet individuals with and without low back pain. J Electromyogr Kinesiol. 2018;39:35-41.

18. Santos F, Carmo C, Fracini A, Pereira R, Takara K, Tanaka C. Chronic low back pain in women: muscle activation during task performance. J Phys Ther Sci. 2013;25(12):1569-73.

19. Moher D, Shamseer L, Clarke M, Ghersi D, Liberati A, Petticrew M, Shekelle P, Stewart L. Preferred reporting items for systematic review and meta-analysis protocols (PRISMA-P) 2015 statement. Syst Rev. 2015;4(1):1.

20. Koes B, van Tulder M, Thomas S. Diagnosis and treatment of low back pain. BMJ. 2006;332(7555):1430-4.

21. Mangum L, Murray K, Saliba S. Mechanical and electrical evaluation of muscle activity during exercise in individuals with and without low back pain. J Athl Train. 2017;52(6):1.

22. Chacon M. The relationship of trunk and hip muscle strength to low back pain. Eugene: Microform Publications, College of Human Development and Performance, University of Oregon; 1990.

23. Cai C, Kong P. Low back and lower-limb muscle performance in male and female recreational runners with chronic low back pain. J Orthop Sports Phys Ther. 2015;45(6):436-43.

24. Embaby E, Abdallah A. Trunk and gluteus-medius muscles' fatigability during occupational standing in clinical instructors with low back pain. Int J Med Health Pharm Biomed Eng. 2014;8(3):169-74.

25. Hides J, Oostenbroek T, Franettovich Smith M, Smith N, Mendis D. The effect of low back pain on trunk muscle size/function and hip strength in elite soccer players. J Sci Med Spor. 2016;20:e87.

26. Hungerford B, Gilleard W, Hodges P. Evidence of altered lumbopelvic muscle recruitment in the presence of sacroiliac joint pain. Spine (Phila Pa 1976). 2003;28(14):1593-600.

27. Iglesias-Gonzalez J, Munoz-Garcia M, Rodrigues-de-Souza DP, AlburquerqueSendin F, Fernandez-de-las-Penas C. Myofascial trigger points, pain, disability, and sleep quality in patients with chronic nonspecific low back pain. Pain Med. 2013;14(12):1964-70.

28. Notzel D, Puta C, Wagner H, Anders C, Petrovich A, Gabriel H. Altered hip muscle activation in patients with chronic non-specific low back pain. Schmerz (Berlin, Germany). 2011;25(2):199-204 206.

29. Penney T, Ploughman M, Austin MW, Behm DG, Byrne JM. Determining the activation of gluteus medius and the validity of the single leg stance test in chronic, nonspecific low back pain. Arch Phys Med Rehabil. 2014;95(10): 1969-76.

30. Rabel M, Chase R, Lominac M, Yoon C. Muscle timing and activation patterns during two movement screening tests in subjects with and without low back pain. J Nat Soc Allied Health. 2013;10(1):38-48.
31. Ringheim I, Austein H, Indahl A, Roeleveld K. Postural strategy and trunk muscle activation during prolonged standing in chronic low back pain patients. Gait Posture. 2015;42(4):584-9.

32. Skorupska E, Keczmer P, Lochowski R, Tomal P, Rychlik M, Samborski W. Reliability of MR-based volumetric 3-D analysis of pelvic muscles among subjects with low back with leg pain and healthy volunteers. PLoS One. 2016;11:7.

33. Sutherlin $\mathrm{M}$, Hart J. Hip-abduction torque and muscle activation in people with low back pain. J Sport Rehabil. 2015;24(1):51-61.

34. Sutherlin M, Hart J. Hip function and predictors of subjective outcomes in individuals with a history of low back pain. Athletic Training Sports Health Care. 2015b;7(3):108-16.

35. Farasyn A, Meeusen R. The influence of non-specific low back pain on pressure pain thresholds and disability. Eur J Pain Title. 2005;9(4):375-81.

36. Njoo K, Van der Does E. The occurrence and inter-rater reliability of myofascial trigger points in the quadratus lumborum and gluteus medius: a prospective study in non-specific low back pain patients and controls in general practice. Pain. 1994;58(3):317-23.

37. Mendis MD, Hides JA. Effect of motor control training on hip muscles in elite football players with and without low back pain. J Sci Med Sport. 2016; 19(11):866-71.

38. Larsen LH, Hirata RP, Graven-Nielsen T. Experimental low Back pain decreased trunk muscle activity in currently asymptomatic recurrent low Back pain patients during step tasks. J Pain. 2018:19(5):542-51.

39. Aboufazeli M, Akbari M, Jamshidi AA, Jafarpisheh MS. Comparison of selective local and global muscle thicknesses in females with and without chronic low Back pain. Ortop Traumatol Rehabil. 2018;20(3):197-204.

40. Nadler S, Malanga G, DePrince M, Stitik T, Feinberg J. The relationship between lower extremity injury, low back pain, and hip muscle strength in male and female collegiate athletes. Clin J Sport Med. 2000;10(2):89-97.

41. Neumann DA. Kinesiology of the hip: a focus on muscular actions. J Orthop Sports Phys Ther. 2010;40(2):82-94.

42. Trendelenburg F. Trendelenburg's test. Clin Orthop Relat Res. 1895; 1998(355):3-7.

43. Fujita K, Kabata T, Kajino Y, Iwai S, Kuroda K, Hasegawa K, Fujiwara K, Tsuchiya H. Quantitative analysis of the Trendelenburg test and invention of a modified method. J Orthop Sci. 2017;22(1):81-8.

44. Willson JD, Dougherty CP, Ireland ML, McClay Davis I. Core stability and its relationship to lower extremity function. J Am Acad Orthop Surg. 2005; 13(5):316-25.

45. Christopher $P$. The influence of abnormal hip mechanics on knee injury: a biomechanical perspective. J Orthop Sports Phys Ther. 2010;40(2):42-51.

46. Khamis S, Dar G, Peretz C, Yizhar Z. The relationship between foot and pelvic alignment while standing. J Hum Kinet. 2015;46:85-97.

47. Lucas $K$. The impact of latent trigger points on regional muscle function. Curr Pain Headache Rep. 2008;12(5):344-9.

48. Amundsen PA, Evans DW, Rajendran D, Bright P, Bjørkli T, Eldridge $S$, Buchbinder R, Underwood M, Froud R. Inclusion and exclusion criteria used in non-specific low back pain trials: a review of randomised controlled trials published between 2006 and 2012. BMC Musculoskelet Disord. 2018;19(1):113.

\section{Publisher's Note}

Springer Nature remains neutral with regard to jurisdictional claims in published maps and institutional affiliations.

Ready to submit your research? Choose BMC and benefit from:

- fast, convenient online submission

- thorough peer review by experienced researchers in your field

- rapid publication on acceptance

- support for research data, including large and complex data types

- gold Open Access which fosters wider collaboration and increased citations

- maximum visibility for your research: over $100 \mathrm{M}$ website views per year

At BMC, research is always in progress.

Learn more biomedcentral.com/submissions 